\title{
Atuação do enfermeiro no cuidado humanizado ao idoso na Atenção Primária
}

\author{
Nurse's role in humanized care for the elderly in Primary Care \\ Papel de la enfermera en la atención humanizada al anciano en Atención Primaria
}

\author{
Ana Fátima Souza Melo de Andrade \\ ORCID: https://orcid.org/0000-0002-7024-6175 \\ Centro Universitário Estácio de Sergipe, Brasil \\ E-mail: anafatimamelo@ hotmail.com \\ Rebeca Galvão Fonseca \\ ORCID: https://orcid.org/0000-0002-4059-1226 \\ Centro Universitário Estácio de Sergipe, Brasil \\ E-mail: rebeca_galvao@yahoo.com.br \\ Weber de Santana Teles \\ ORCID: https://orcid.org/0000-0003-1770-8278 \\ Centro de Hemoterapia de Sergipe, Brasil \\ E-mail: arteecura@hotmail.com \\ Max Cruz da Silva \\ ORCID: https://orcid.org/0000-0002-6944-5986 \\ Faculdade Pio Décimo, Brasil \\ E-mail: maxlfi@hotmail.com \\ Ângela Maria Melo Sá Barros \\ ORCID: https://orcid.org/0000-0003-4087-3247 \\ Universidade Federal do Rio de Janeiro, Brasil \\ E-mail: angelsamelo@hotmail.com \\ Ruth Cristini Torres \\ ORCID: https://orcid.org/0000-0002-8664-192X \\ Instituto de Hematologia e Hemoterapia de Sergipe, Brasil \\ E-mail: ruthcristini@gmail.com \\ Alejandra Debbo \\ ORCID: https://orcid.org/0000-0002-7743-5921 \\ Universidade Tiradentes, Brasil \\ E-mail: aledebbo@hotmail.com \\ Marcel Vinícius Cunha Azevedo \\ ORCID: https://orcid.org/0000-0002-5312-3333 \\ Centro Universitário Estácio Sergipe, Brasil \\ E-mail: marcelvinicius49@gmail.com \\ Paulo Celso Curvelo Santos Junior \\ ORCID: https://orcid.org/0000-0001-5834-6782 \\ Universidade Tiradentes, Brasil \\ E-mail: paulo.curvelo.jr@gmail.com \\ Maria Hozana Santos Silva \\ ORCID: https://orcid.org/0000-0001-5742-5366 \\ Faculdade Ages de Medicina, Brasil \\ E-mail: hosana_p@hotmail.com \\ Taíssa Alice Soledade Calasans \\ ORCID: https://orcid.org/0000-0003-0460-4437 \\ Universidade Tiradentes, Brasil \\ E-mail: taissa.asc@gmail.com
}

\begin{abstract}
Resumo
Este estudo tem como objetivo: Identificar na literatura a atuação do enfermeiro no cuidado humanizado ao idoso na atenção primária; Verificar os métodos desenvolvidos pelos enfermeiros que propicia um cuidado humanizado ao idoso na atenção primária; Conhecer os benefícios do cuidado humanizado realizado pelo enfermeiro ao idoso. A presente pesquisa utilizou como método a revisão integrativa de literatura, com dados quantitativos, descritivo e exploratório. A seleção dos dados ocorreu no dia 08 de Abril de 2020, através de artigos indexados nas seguintes bases de dados: Base de Dados em Enfermagem (BDENF), Literatura Latino americana e Caribe em ciências da Saúde (LILACS) e Scientific Eletronic Library Online (SciELO). Foram incluídosna pesquisa artigos originais, em português, nacional, disponíveis na íntegra e gratuitos. Foram excluídos teses, recortes de revistas e jornais, cartas ao editor, congresso resumos e metanálises, além dos artigos inferior ao ano de 2014 e que não atenderam aos objetivos e questões estabelecidas. Após triagem, foram selecionados 17 artigos que compuseram aamostra final. Percebeu-se um resultado positivo dos dados, constatando que a atuação do enfermeiro no cenário da atenção primária frente ao cuidado humanizado fundamental.
\end{abstract}


As publicações permitiram a efetivação das variáveis, que foram categorizadas na discussão, sendo elas: A atuação do enfermeiro no cuidado humanizado ao idoso na atenção primária; Métodos desenvolvidos pelos enfermeiros que propicia um cuidado humanizado ao idoso na atenção primária; Benefícios do cuidado humanizado realizado pelo enfermeiro ao idoso. Foi possível compreender a importância da atuação do enfermeiro no cuidado humanizado ao idoso na atenção primária, o enfermeiro é um profissional indispensável nesse contexto, contribuindo positivamente na equipe multiprofissional com ênfase na saúde do idoso.

Palavras-chave: Atenção Primária à Saúde; Cuidados de enfermagem; Enfermagem geriátrica; Saúde do idoso.

\begin{abstract}
This study aims to: Identify, in the literature, the role of nurses in humanized care for the elderly in primary care; Check the methods developed by nurses that provide humanized care for the elderly in primary care; Know the benefits of humanized care provided by nurses to the elderly. This research used the integrative literature review as a method, with quantitative, descriptive and exploratory data. Data selection took place on April 8, 2020, through articles indexed in the following databases: Database in Nursing (BDENF), Latin American and Caribbean Literature in Health Sciences (LILACS) and Scientific Electronic Library Online ( SciELO). Original articles, in Portuguese, national, available in full and free of charge, were included in the research. Theses, clippings from magazines and newspapers, letters to the editor, congress, abstracts and meta-analyses, in addition to articles inferior to the year 2014 and that did not meet the established objectives and issues, were excluded. After screening, 17 articles were selected that made up the final sample. There was a positive result of the data, noting that the role of nurses in the primary care setting in relation to fundamental humanized care. The publications allowed the realization of the variables, which were categorized in the discussion, namely: The role of nurses in humanized care for the elderly in primary care; Methods developed by nurses that provide humanized care for the elderly in primary care; Benefits of humanized care provided by nurses to the elderly. It was possible to understand the importance of the nurse's role in humanized care for the elderly in primary care, the nurse is an indispensable professional in this context, contributing positively to the multidisciplinary team with an emphasis on the health of the elderly.
\end{abstract}

Keywords: Primary Health Care; Nursing care; Geriatric nursing; Elderly health.

\title{
Resumen
}

Este estudio tiene como objetivo: Identificar, en la literatura, el papel del enfermero en la atención humanizada al anciano en la atención primaria; Consultar los métodos desarrollados por enfermeras que brindan atención humanizada al anciano en atención primaria; Conocer los beneficios del cuidado humanizado que brindan las enfermeras a las personas mayores. Esta investigación utilizó como método la revisión integradora de la literatura, con datos cuantitativos, descriptivos y exploratorios. La selección de datos se realizó el 8 de abril de 2020, a través de artículos indexados en las siguientes bases de datos: Base de Datos en Enfermería (BDENF), Literatura Latinoamericana y del Caribe en Ciencias de la Salud (LILACS) y Biblioteca Electrónica Científica en Línea (SciELO). En la investigación se incluyeron artículos originales, en portugués, nacionales, disponibles en su totalidad y de forma gratuita. Se excluyeron tesis, recortes de revistas y periódicos, cartas al editor, congresos, resúmenes y metaanálisis, además de artículos inferiores al año 2014 y que no cumplieran con los objetivos y temas establecidos. Después del cribado, se seleccionaron 17 artículos que conformaron la muestra final. Hubo un resultado positivo de los datos, destacando que el papel de las enfermeras en el ámbito de la atención primaria en relación con la atención humanizada fundamental. Las publicaciones permitieron la concreción de las variables, que fueron categorizadas en la discusión, a saber: El rol del enfermero en la atención humanizada al anciano en la atención primaria; Métodos desarrollados por enfermeras que brindan atención humanizada al anciano en atención primaria; Beneficios de la atención humanizada brindada por enfermeras a las personas mayores. Se pudo comprender la importancia del rol de la enfermera en la atención humanizada al anciano en la atención primaria, la enfermera es un profesional indispensable en este contexto, contribuyendo positivamente al equipo multidisciplinario con énfasis en la salud del anciano.

Palabras clave: Primeros Auxilios; Cuidado de enfermera; Enfermería geriátrica; Salud de los ancianos.

\section{Introdução}

No Brasil, houve uma grande mudança demográfica, principalmente desde as últimas décadas do milênio passado, aumentando o número da população idosa brasileira, passandode 7 milhões na década de 1980 para 11 milhões na década de 1990. (Sanguino, 2018).

O envelhecer não significa estar ou ser doente, mas que necessita de cuidados devido às mudanças ao passar dos anos. No entanto, verifica-se que a elaboração de estratégias é um dos grandes desafios a serem enfrentados pelas Políticas de Saúde Pública, essas ações dependem de um olhar integral para promover a qualidade de vida, sendo fundamental o cuidado de enfermagem para seguir uma linha de cuidado (Santos, 2016). 
A Política Nacional de Humanização (PNH) vem para efetivar os princípios do SUS no cotidiano da atenção à saúde e dar voz aos usuários, aos gestores e aos profissionais da saúde com a finalidade de melhoria do processo e da integração dos mesmos, o acolhimento emerge como uma ferramenta eficaz dessa política (Maciel, Silva \& Oliveira, 2019).

Frente aos programas para o processo de humanização, a Política Nacional de Humanização (PNH) que recomenda que o acolhimento deve estar sempre presente nos momentos do processo de atenção à saúde, apesar de estar incluída em todas as relações humanas. Incluindo a Política Nacional do Idoso (PNI) revelando em seu art. $2^{\circ}$, direitos para garantir a preservação da saúde física e mental, o aperfeiçoamento moral, intelectual, espiritual e social para dignidade do idoso (Silva, 2018).

Nessa perspectiva, o idoso precisa ser incentivado para a adesão da qualidade de vida, o atendimento humanizado de enfermagem contribue para essa finalidade, sendo que a atenção primáriadeve ser o contato preferencial dos idosos, tornandose a principal porta de entrada para a atenção em saúde, envolvendo-os nas atividades em prol do seu cuidado (Hoepers, 2016).

Os profissionais de enfermagem que atuam na atenção primária recomendam sugestões para melhorar a assistência ao idoso, como cultivar alianças com os familiares para o fortalecimento do cuidado, realizar adequações quanto ao dimensionamento de pessoal de enfermagem devido grau elevado de dependência dos pacientes idosos (Nascimento, 2015).

Portanto, a literatura revela a impotância do cuidado humanizado ao paciente idoso assistido na atenção primária. Humanizar em saúde é um processo complexo, abrangendo protótipos diferenciados na visão dos trabalhadores, mediante crenças e valores, ao refletir sobre suas ações de cuidado e a humanização da assistência(Oliveira, 2018).

Diante do exposto, surgiu uma questão, norteadora elaborada a partir da estratégia PICO que significa: P (População Alvo) I (Área de Interesse), C (Contexto) O (Desfecho) assim como o nível de evidência, a questão foi: Quais os benefícios para a população idosa por meio do cuidado humanizado?. Esse estudo justifica-se por ser de grande valia o cuidado humanizado, principalmente ao paciente idoso assistido na atenção primária, onde a atuação humanizada do enfermeiro nesse cenário é fundamental por ser uma profissão relacionada ao cuidado direto e contínuo, além de promover benefícios aos pacientes idosos por meio do cuidado individualizado, esses profissionais obtém resultados positivos em sua assistência, gerando benefícios para essa comunidade assistida.

Os objetivos do estudo foram, Identificar na literatura a atuação do enfermeiro no cuidado humanizado ao idoso na atenção primária; Verificar os métodos desenvolvidos pelos enfermeiros que propicia um cuidado humanizado ao idoso na atenção primária; Conhecer os benefícios do cuidado humanizado realizado pelo enfermeiro ao idoso.

\section{Metodologia}

A presente pesquisa utilizou como método a revisão integrativa de literatura, com dados quantitativos, descritivo e exploratório, por meio de buscas em publicações científicas para contribuição e efetivação da temática abordada. O estudo de revisão integrativa é a base para a identificação do atual conhecimento científico, parte-se dela para buscar hiatos a serem explorados em determinadas temáticas, sendo na revisão que o pesquisador se familiariza comos textos, identifica os autores que vêm escrevendo sobre o problema pesquisado(Ferenhof \& Fernandes, 2016).

A primeira etapa da pesquisa foi composta pela delimitação do tema, sendo este: Atuação do enfermeiro no cuidado humanizado ao idoso na atenção primária, na intenção de responder as seguintes questões norteadoras: Como vem sendo desenvolvida a atuação do enfermeiro no cuidado humanizado ao idoso na atenção primária? Quais os métodos desenvolvidos pelos enfermeiros que propicia um cuidado humanizado ao idoso na atenção primária? Quais os benefícios do cuidado humanizado realizado pelo enfermeiro ao idoso?.

Para efetivação da segunda etapa dessa pesquisa, a seleção dos dados ocorreu no dia 08 de Abril de 2020, através de artigos científicos indexados nas seguintes bases de dados: Base de Dados em Enfermagem (BDENF), Literatura Latino americana e Caribe em ciências da Saúde (LILACS) e Scientific Eletronic Library Online (SciELO). Os achados foram 
levantados a partir de consultas ao Descritores em Ciências da Saúde (DeCS): Atençãoprimária à Saúde; Assistência de Enfermagem; Enfermagem geriátrica; Humanização; Saúde do idoso, o o auxilio do operador booleano AND.

Para contribuir no desenvolvimento do estudo, na terceira etapa foram elencadosalguns critérios para a seleção da amostra, foram incluídos na pesquisa artigos em português, nacional, disponíveis na íntegra e gratuitos. Foram excluídos teses, recortes de revistas e jornais, cartas ao editor, congresso resumos, revisões sistemáticas e metanálises, além dos artigos inferior ao ano de 2014 e que não atenderam aos objetivos e questões estabelecidas nessa pesquisa.

A quarta etapa houve a seleção dos artigos da pesquisa, sendo identificados 125 artigos relacionados ao tema estudado, posteriormente, houve uma leitura analítica inicial dos resumos dos artigos, os que atenderam aos critérios estabelecidos foram explorados em textos completos, que permitiu verificar detalhadamente os achados. Em seguida, resultando na exclusão de 108 artigos que não foram pernitentes aos objetivos e questões elaboradas. Após triagem, foram selecionados 17 artigos que preencheram aos critérios de inclusão, e compuseram a amostra final deste estudo.

Na quinta etapa, houve a elaboração de quatro quadros nos resultados para apresentar os artigos incluídos no estudo. O Quadro 1 foi composto por características primárias dos estudos incluídos na pesquisa: Título, Autores, Periódico / Ano e Objetivos do estudo, que permitiram destacar todos os artigos selecionados. O Quadro 2 revelando os achados daprimeira categoria, o Quadro 3 com os dados referente ao conteúdo da segunda categoria, e o Quadro 4 destacando os dados da terceira categoria.

Na sexta etapa, na tentativa de responder os objetivos e questões norteadoras, foram elaboradas três categorias, a amostra foi distribuídas na discussão de acordo com as variáveis, sendo elas: Atuação do enfermeiro no cuidado humanizado ao idoso na atenção primária; Métodos desenvolvidos pelos enfermeiros que propicia um cuidado humanizado ao idoso na atenção primária; Benefícios do cuidado humanizado realizado pelo enfermeiro ao idoso.

Desta forma, os dados selecionados das publicações incluídas no estudo foram respeitandas integralmente nos resultados e discussão desse estudo, identificando o autor confome apresentações dos conteúdos, como preconiza a Lei 12.853 de 14 de Agosto do ano 2013, segundo seu Art. 1º., Lei que regula os direitos autorais, onde entendendo-se sob esta denominação os direitos de autor e os que lhes são conexos.

\section{Resultados}

Demonstrou-se nos resultados deste estudo os conhecimentos sobre o enfermeiro no cuidado humanizado ao idoso na atenção primária. Dentre os artigos selecionados, houve a elaboração de quadros para apresentar os resultados dessa pesquisa. Inicialmente, foi elaborado um quadro sintético composto por características primárias de todos os estudos incluídos na discussão, os mesmos apresentaram dados fundamentais, com tópicos releventes. Com a inclusão desses estudos foi possível discutir e comparar com outras literaturas para compreender como as presentes variáveis estão sendo abordadas no meio científico. O Quadro 1 foi construído para revelar os tópicos dos 17 artigos que compuseram a amostra final. 
Quadro 1. Características primárias dos estudos incluídos na pesquisa, Aracaju, SE, 2020.

\begin{tabular}{|c|c|c|c|}
\hline Título & Autores & Periódico / Ano & Objetivo do estudo \\
\hline $\begin{array}{l}\text { Discursos dos profissionais de } \\
\text { saúde da família na ótica da } \\
\text { assistência à saúde do idoso }\end{array}$ & $\begin{array}{l}\text { Amthauer, C., \& } \\
\text { Falk, J.W. }\end{array}$ & $\begin{array}{l}\text { Revista Online de } \\
\text { Pesquisa Cuidado é } \\
\text { fundamental / } 2017\end{array}$ & $\begin{array}{l}\text { Identificar as práticas realizadas pelos } \\
\text { profissionais da Estratégia de Saúde da Família } \\
\text { (ESF) junto aos idosos atendidos em uma } \\
\text { Unidade Básica de Saúde (UBS) }\end{array}$ \\
\hline $\begin{array}{l}\text { Avaliação da atençãointegral à } \\
\text { saúde do idoso na percepção } \\
\text { deprofissionais }\end{array}$ & $\begin{array}{l}\text { Cabral, J.F., et } \\
\text { al. }\end{array}$ & $\begin{array}{l}\text { Revista Brasileira em } \\
\text { Promoção da Saúde / } \\
2019\end{array}$ & $\begin{array}{l}\text { Avaliar a atenção integral à saúde doidoso } \\
\text { segundo a percepção de profissionais em } \\
\text { Unidades de Saúde daFamília (USF) }\end{array}$ \\
\hline $\begin{array}{l}\text { Subconjunto de Diagnósticos de } \\
\text { Enfermagem para idosos na } \\
\text { AtençãoPrimária à Saúde }\end{array}$ & $\begin{array}{l}\text { Clares, J.W.B., } \\
\text { et al. }\end{array}$ & $\begin{array}{l}\text { Revista da Escola de } \\
\text { Enfermagem da USP / } \\
2016\end{array}$ & $\begin{array}{l}\text { Desenvolver diagnósticos de enfermagem para } \\
\text { idosos acompanhados na Atenção Primária à } \\
\text { Saúde, com base no banco de termos para a } \\
\text { prática enfermagem com idosos }\end{array}$ \\
\hline $\begin{array}{l}\text { Influência da enfermagem na } \\
\text { adesão do idoso ao tratamento } \\
\text { da hipertensão }\end{array}$ & $\begin{array}{l}\text { Dias, E.G., Souza, } \\
\text { E.L.S., \&Mishima, } \\
\text { S.M. }\end{array}$ & $\begin{array}{l}\text { Revista Eletrônica } \\
\text { Gestão \& Saúde / } 2016\end{array}$ & $\begin{array}{l}\text { Investigar a influencia da Enfermagem na } \\
\text { adesão do idoso ao tratamento da hipertensão }\end{array}$ \\
\hline $\begin{array}{l}\text { Estrutura conceptual do } \\
\text { envelhecimento em diferentes } \\
\text { etnias }\end{array}$ & $\begin{array}{l}\text { FAller, J.W., } \\
\text { Teston, E.F.;, \& } \\
\text { Marcon, S.S. }\end{array}$ & $\begin{array}{l}\text { Revista Gaúcha de } \\
\text { Enfermagem / } 2018\end{array}$ & $\begin{array}{l}\text { Elaborar uma estrutura que expliquecomo } \\
\text { idosos de diferentes culturas vivenciam o } \\
\text { envelhecimento }\end{array}$ \\
\hline $\begin{array}{l}\text { Melhores práticas de } \\
\text { enfermagem na saúde da pessoa } \\
\text { idosa }\end{array}$ & $\begin{array}{l}\text { Freitas, M.A., \& } \\
\text { Alvarez, A.M. }\end{array}$ & $\begin{array}{l}\text { Revista de } \\
\text { Enfermagem UFPE } \\
\text { Online / } 2020\end{array}$ & $\begin{array}{l}\text { Compreender as experiências de busca por } \\
\text { conhecimento e utilização da experiência } \\
\text { profissional dos enfermeiros no cuidado da } \\
\text { pessoa idosana Atenção Primária à Saúde }\end{array}$ \\
\hline $\begin{array}{l}\text { Humanização naatenção básica } \\
\text { de saúde na percepção de idosos }\end{array}$ & $\begin{array}{l}\text { Lima, T.J.V., et } \\
\text { al. }\end{array}$ & $\begin{array}{l}\text { Saúde e Sociedade / } \\
2014\end{array}$ & $\begin{array}{l}\text { Analisar a percepção do idoso quantoao } \\
\text { cuidado humanizado na atenção básica de } \\
\text { saúde }\end{array}$ \\
\hline $\begin{array}{l}\text { A perspectiva do usuário na } \\
\text { atenção básica sobre } \\
\text { acolhimento ao idoso }\end{array}$ & Medeiros, C.B., et al. & $\begin{array}{l}\text { Revista Ciência } \\
\text { Plural / } 2018\end{array}$ & $\begin{array}{l}\text { Analisou a perspectiva do usuário na Atenção } \\
\text { Básica sobre o acolhimento aoidoso }\end{array}$ \\
\hline $\begin{array}{l}\text { Violência contra idosos: } \\
\text { concepções dosprofissionais de } \\
\text { enfermagem acerca da detecção } \\
\text { e prevenção }\end{array}$ & $\begin{array}{l}\text { Oliveira, K.S.M., et } \\
\text { al. }\end{array}$ & $\begin{array}{l}\text { Revista Gaúcha de } \\
\text { Enfermagem / } 2018\end{array}$ & $\begin{array}{l}\text { Analisar as concepções dos profissionais de } \\
\text { enfermagem atuantesem Unidades Básicas de } \\
\text { Saúde quanto à detecção e prevenção de idosos } \\
\text { violentados }\end{array}$ \\
\hline $\begin{array}{l}\text { Tecnologias na promoção da } \\
\text { saúde de idosos com doenças } \\
\text { crônicas na atenção } \\
\text { primária }\end{array}$ & $\begin{array}{l}\text { Penha, A.A.G., } \\
\text { et al. }\end{array}$ & $\begin{array}{l}\text { Revista de } \\
\text { Enfermagem da } \\
\text { UFSM / } 2015\end{array}$ & $\begin{array}{l}\text { Conhecer as tecnologias empregadas pelos } \\
\text { enfermeiros na promoção da saúde de idosos } \\
\text { com doenças crônicas na Atenção Primária à } \\
\text { Saúde }\end{array}$ \\
\hline $\begin{array}{l}\text { Implantação da Sistematização } \\
\text { da Assistência de Enfermagem } \\
\text { como Qualificação daassistência } \\
\text { ao idoso }\end{array}$ & $\begin{array}{l}\text { Piccinini, V.M., } \\
\text { Costa, } \\
\text { A.E.K., \& Pissaia, } \\
\text { L.P. }\end{array}$ & $\begin{array}{l}\text { Revista Brasileira de } \\
\text { Ciências do } \\
\text { Envelhecimento } / 2017\end{array}$ & $\begin{array}{l}\text { Descrever o processo de implantação da } \\
\text { Sistematização da Assistência de Enfermagem } \\
\text { como meio de qualificação da assistência ao } \\
\text { idoso na Atenção Primária }\end{array}$ \\
\hline $\begin{array}{ll}\text { Idosos atendidos em unidade de } \\
\text { pronto- } & \text { atendimento por } \\
\text { condições } & \text { sensíveis à atenção } \\
\text { primária } & \end{array}$ & $\begin{array}{l}\text { Rissardo, L.K., } \\
\text { et al. }\end{array}$ & $\begin{array}{l}\text { Revista Mineira de } \\
\text { Enfermagem / } 2016\end{array}$ & $\begin{array}{l}\text { Identificar os motivos que levam idosos em } \\
\text { condições sensíveis àatenção primária à saúde }\end{array}$ \\
\hline $\begin{array}{l}\text { Percepção deenfermeiros sobre } \\
\text { o atendimento à pessoa idosa } \\
\text { em contexto de atenção primária }\end{array}$ & $\begin{array}{l}\text { Rodrigues, } \\
\text { A.R.S., et al. }\end{array}$ & $\begin{array}{l}\text { Enfermagem Brasil } \\
\text { / } 2018\end{array}$ & $\begin{array}{l}\text { Compreender a percepção dosenfermeiros sobre } \\
\text { o atendimento ao usuário idoso na Unidade } \\
\text { Básica de Saúde }\end{array}$ \\
\hline
\end{tabular}




\begin{tabular}{|c|c|c|c|}
\hline $\begin{array}{l}\text { Percepção dos idosos acerca da } \\
\text { assistência humanizada de } \\
\text { enfermagem frente ao mal de } \\
\text { Parkinson }\end{array}$ & $\begin{array}{l}\text { Rodrigues, } \\
\text { W.P. et al. }\end{array}$ & $\begin{array}{l}\text { Brazilian Journal of } \\
\text { health Review / } 2019\end{array}$ & $\begin{array}{l}\text { Avaliar a importância do atendimento } \\
\text { humanizado em idosos portadores do mal de } \\
\text { Parkinson através de uma assistência de } \\
\text { enfermagem }\end{array}$ \\
\hline $\begin{array}{l}\text { Avanços e desafios da atenção à } \\
\text { saúde da população idosa com } \\
\text { doenças crônicas na Atenção } \\
\text { Primária }\end{array}$ & $\begin{array}{l}\text { Schenker, M., \& } \\
\text { Costa, D.H. }\end{array}$ & $\begin{array}{l}\text { Ciência \& Saúde } \\
\text { Coletiva / } 2019\end{array}$ & $\begin{array}{l}\text { Analisar os avanços e os desafios da atenção à } \\
\text { saúde da população idosa, sobretudo daquela } \\
\text { com doençascrônicas na atenção primária }\end{array}$ \\
\hline $\begin{array}{l}\text { Capacidade funcional de idosos } \\
\text { atendidos pelo programa saúde } \\
\text { dafamília }\end{array}$ & $\begin{array}{l}\text { Sousa, F.J.D., } \\
\text { Golçalves, } \\
\text { L.H.T., \& Gamba, } \\
\text { M.A. }\end{array}$ & $\begin{array}{c}\text { Revista Cuidarte / } \\
2018\end{array}$ & $\begin{array}{l}\text { Descrever a capacidade funcional de idosos } \\
\text { atendidos pelo programa saúdeda família da } \\
\text { região Norte do Brasil }\end{array}$ \\
\hline $\begin{array}{l}\text { Acesso à Atenção Primária à } \\
\text { Saúde delongevos: perspectiva } \\
\text { de profissionais da Saúde da } \\
\text { Família de um município do } \\
\text { Rio Grande do Sul }\end{array}$ & $\begin{array}{l}\text { Sulzbach, C.C., } \\
\text { Weiller,T.H., \& } \\
\text { Dallepiane, L.B. }\end{array}$ & $\begin{array}{l}\text { Cadernos de Saúde } \\
\text { Coletiva / } 2020\end{array}$ & $\begin{array}{l}\text { Analisar a percepção sobre o acesso de longevos } \\
\text { à Atenção Primária à Saúde na perspectiva dos } \\
\text { profissionais da Estratégia Saúde da Família, em } \\
\text { ummunicípio do Sul do Brasil }\end{array}$ \\
\hline
\end{tabular}

Fonte: Elaboração própria.

Com base no cenário da atenção primária foi construído o Quadro 2 para destacar as principais representações dos autores referente a primeira categoria da discussão. Desses, 6 artigos revelam as seguintes representações: As alternativas para a atenção integral à saúde doidoso; Fundamental importância dos enfermeiros nas estratégias de prevenção, monitorização e controle de doenças; Medidas dos profissionais para tornar mínimo os problemas da população idosa; O enfermeiro é referência na equipe multiprofissional; Preparo para atuar com base nos princípios do SUS; O enfermeiro tem competências e autonomia.

Quadro 2. Atuação do enfermeiro na atenção primária, segundo Autor/ano, Aracaju, SE,2020.

\begin{tabular}{|l|c|}
\hline \multicolumn{1}{|c|}{ Atuação do enfermeiro na atenção primária } & Autores / Ano \\
\hline Alternativas para a atenção integral à saúde do idoso & Cabral et al. / 2019 \\
\hline $\begin{array}{l}\text { Fundamental importância dos enfermeiros nas estratégias de prevenção, } \\
\text { monitorização e controle de doenças }\end{array}$ & Dias, Souza e Mishima /2016 \\
\hline Medidas dos profissionais para tornar mínimo os problemas da população idosa & Faller, Teston e Marcon / \\
\hline O enfermeiro é referência na equipe multiprofissional & Freitas e Alvarez / 2020 \\
\hline É fundamental o preparo para atuar com base nos princípios do SUS & Medeiros et al. / 2018 \\
\hline O enfermeiro tem competências e autonomia & Rissardo et al. / 2016 \\
\hline
\end{tabular}

Fonte: Elaboração própria.

Conforme a segunda categoria do estudo, identificou-se 6 Métodos que propiciam um cuidado humanizado, sendo eles: Diálogo; linguagem clara; tratamento individualizado epersonalizado; acolhimento, confiança e respeito; Sistematização da Assistência de Enfermagem (SAE) e vínculo; Empatia; carinho; Respeito e Comunicar-se com linguagem acessível e Criação de vínculo; Respeito; Confiança e uso de práticas não invasivas, como demonstrado no Quadro 3. 
Quadro 3. Pincipais métodos que propicia um cuidado humanizado, segundo Autor/ano,Aracaju, SE, 2020.

\begin{tabular}{|c|c|}
\hline Métodos que propicia um cuidado humanizado & Autores / Ano \\
\hline Diálogo; linguagem clara; tratamento individualizado e personalizado & Dias, Souza e Mishima /2016 \\
\hline Acolhimento & Medeiros et al. / 2018 \\
\hline Confiança e respeito & Oliveira et al. / 2018 \\
\hline Sistematização da Assistência de Enfermagem (SAE) e vínculo & Piccinin, Costa e Pissaia \\
\hline Empatia; carinho; respeito e comunicar-se com linguagem acessível & Rodrigues et al. / 2018 \\
\hline Criação de vínculo; Respeito; confiança e uso de práticas não invasivas & Rodrigues et al. / 2019 \\
\hline
\end{tabular}

Fonte: Elaboração própria.

De acordo com os benefícios para o idoso diante do cuidado humanizado pelo enfermeiro que foram elencados na terceira categoria da discussão o Quadro 4 demostra que amaioria dos estudos revelou sobre a promoção da saúde, dos 6 artigos discutidos. Seguidos dos seguintes benefícios: Protagonismo e corresponsabilidade; Interesse pela vida; Bem-estar físico, mental, social e tranquilidade e Promoção da saúde.

Quadro 4. Benefícios do cuidado humanizado pelo enfermeiro ao idoso, segundo Autor/ano,Aracaju, SE, 2020.

\begin{tabular}{|l|c|}
\hline \multicolumn{1}{|c|}{ Benefícios para o idoso } & Autores / Ano \\
\hline Interesse pela vida, bem-estar físico, mental, social e tranquilidade & Clares et al. / 2016 \\
\hline Protagonismo e corresponsabilidade & Lima et al. / 2014 \\
\hline Promoção da saúde & Penha et al. / 2015 \\
\hline Promoção da saúde & Rodrigues et al. / 2018 \\
\hline Promoção da saúde & Schenker e Costa / 2019 \\
\hline Promoção da saúde & $\begin{array}{c}\text { Sousa, Golçalves e } \\
\text { Gamba / 2018 }\end{array}$ \\
\hline
\end{tabular}

Fonte: Elaboração própria.

\section{Discussão}

As 17 publicações incluídas no presente estudo permitiram a efetivação das variáveis elaboradas que foram categorizadas, sendo elas: A atuação do enfermeiro no cuidado humanizado ao idoso na atenção primária; Métodos desenvolvidos pelos enfermeiros que propicia um cuidado humanizado ao idoso na atenção primária; Benefícios do cuidado humanizado realizado pelo enfermeiro ao idoso. Contribuindo para o desfecho dessa revisão integrativa, atendendo aos resultados esperados.

Categoria 1: Atuação do enfermeiro no cuidado humanizado ao idoso na atenção primária

Ao explorar o estudo de Faller, Teston e Marcon (2018) foi possível identificar que o envelhecer acelerado populacional ocasiona vários problemas sociais, econômicos e de saúde pública, exigindo medidas dos profissionais da atenção primária, 
como os enfermeiros, para tornar mínimo ou até mesmo resolver esses problemas. Entende-se que ao conhecer as particularidades dessa população coopera no desenvolvimento de ações e intervenções diante da necessidade de cada indivíduo e situação.

O Sistema Único de Saúde (SUS) promove a inclusão social no país diante da política dos direitos da população, que fundamenta-se pelos princípios doutrinários que conferem universalidade, integralidade e equidade. Torna-se fundamental no cenário contemporâneo, que estabeleçam alternativas para uma atenção integral à saúde do idoso na atenção primária,a idade traz consigo mudanças que devem ser foco no atendimento humanizado pelos enfermeiros que proporcionam uma atuação de qualidade (Cabral, 2019).

O enfermeiro é um profissional referência na equipe multiprofissional na atenção primária à saúde, por promover uma atuação segura e eficaz, sendo um fio condutor do cuidado em enfermagem. Confia-se que o papel do enfermeiro proporciona o fortalecimento da atenção primária, onde os usuáriosidosos estão inseridos, esse profissionais estão sempre em busca por conhecimento e otimizando o uso de sua experiência para um cuidado sistematizado (Freitas \& Alvarez, 2020).

O enfermeiro tem autonomia e competência para serem agentes de transformação, uma vez que a educação em saúde faz parte das suas atribuições, além da gestão e organização dos atendimentos e orientações para usuários da sua área. Por sua vez, como líder da atenção primária, o enfermeiro deve enfatizar o trabalho no cuidado ao idoso, juntamente com o agente de saúde, pois esse profissional é fundamental no elo entre a unidade e a população,

contribuindo na articulação das informações e na melhor visão dos profissionais de saúde sobre a população (Rissardo, 2016).

A presença dos idosos nos serviços de saúde é fundamental, e os enfermeiros devem ser qualificados para recebê-los da melhor forma, sempre embasados nos princípios e diretrizes do (SUS), como a integralidade, equidade e descentralização, bem como ao uso das políticas de saúde capazes de contribuir com o envelhecimento ativo e saudável, proporcionando um ambiente social e cultural mais favorável para a população idosa sob o acompanhamento na atenção primária (Medeiros, 2018).

Os profissionais que atuam na atenção primária, sobretudo os enfermeiros, desenvolvem um papel de fundamental importância nas estratégias de prevenção, diagnóstico,monitorização, controle de doenças, e adesão ao tratamento para prevenção de complicações caracterizada pela conduta dos assistidos em relação ao hábito de usar medicamentos mediante prescrição, seguindo as mudanças no estilo de vida preconizadas e comparecendo nas consultas (Dias, Souza \& Mishima, 2016).

Na realidade demográfica e epidemiológica que nos está imposta dos dias de hoje, é importante atentar para a urgência de mudanças e inovação nos paradigmas do cuidado à saúde do idoso, com o planejamentos e ações diferenciadas para que os serviços de saúde sejam eficazes em suas ações e que o idoso seja atendido de forma integral e humanizada (Amthauer \& Falk, 2017).

No estudo de Sulzbach, Weiller e Dallepiane, (2020) foi possível perceber que os usuários idosos constituem em uma população mais vulnerável, necessitando de um cuidado diferenciado, com isso, demanda maiores cuidados e torna-se fundamental ampliar o acesso e tecer uma rede de cuidado pautada na humanização.

Diante do exposto, percebeu-se resultados positivos frente aos dados explanados,sinalizando concordâncias entre as variáveis elencadas, constatando que a atuação doenfermeiro no cenário da atenção primária frente ao cuidado humanizado ao idoso é defundamental importância para essa população. Os dados revelaram que o enfermeirodesenvolve um cuidado indispensável na atenção primária, permitindo verificar que a partirdo envelhecimento da população surgem necessidades e problemas que requer uma atençãoespecial, sobretudo os problemas de saúde.

Complementando ao que fo abordado, Silva, et al. (2018) ressalta que na atenção primária, os profissionais da enfermagem oferece uma assistência integral para todos osmembros das famílias com vínculo à unidade, que deve ficar atento às mudanças do perfil populacional, com atenção especial ao idoso para promover uma participação ativa na melhoria de sua qualidade de vida, por meio da promoção, proteção, identificar possíveis agravos, intervenção e medidas de reabilitação. 
Categoria 2: Métodos desenvolvidos pelos enfermeiros que propicia um cuidado humanizadoao idoso na atenção primária

Entende-se que o cuidado humanizado é essencial para a efetivação de uma atuação dequalidade. No estudo de Oliveira, et al. (2018) identificou-se que o enfermeiro no cenário da atenção primária promove suporte para a família e, por conseguinte, o cuidado aos idosos, torna-se responsabilidade desses profissionais favorecer uma atmosfera de confiança paracom o idoso, respeitando suas decisões, oferecer o cuidado ao outro é uma trabalho complexo que vai além do estabelecimento de protocolos e condutas técnicas.

O acolhimento é uma prática fundamental na humanização em saúde, na (PNH), o Ministério da Saúde reforça a valorização dos profissionais que contribuem na promoção da saúde. É perceptível que o cuidado humanizado é constituído por dar atenção especial, ter responsabilidade por alguém, ao passar dos anos esse termo tem se tornado mais voltado para atenção à saúde, ocorrendo principalmente pelo fato do ato de cuidar, o diagnosticar, tratar e prevenir através do conhecimento técnico e científico (Medeiros, 2018).

Ao identificar os achados no estudo de Rodrigues, et al. (2018) demosntrou que na consulta de enfermagem como prática já integralizada no cotidiano de trabalho dos enfermeiros na atenção primária, promove o desenvolvimento de práticas de cuidados envolvendo o fortalecimento de vínculos. A conduta humanizada são evidenciadas em suas falas durante essa pesquisa, relatando uma preocupação com os usuários idosos, não apenas na consulta, mas quando procuram saber sobre o seu ambiente familiar. Frente aos relatos, percebeu-se sentimentos de empatia, carinho e respeito, bem como a utilização de uma linguagem acessível ao idoso durante os atendimentos.

A atuação dos profissionais da enfermagem diante da influência para adesão ao tratamento de doeças, os idosos relatam a importância da atuação da enfermagem, principalmente quando é realizado de forma humanizada. A adesão está fortemente associadaà relação dos profissionais de saúde com o usuário, sendo necessário que o diálogo por meio de uma linguagem clara, tratamento individualizado e personalizado, respeitando suas capacidades cognitivas e situação socioeconômica (Dias, Souza \& Mishima, 2016).

Para compreender as diversas formas de humanização, o estudo de Peccinin, Costa e Pissaia, (2017) complementam que a qualificação da assistência vem sendo percebida pelos profissionais como um diferencial na atuação de enfermagem com o uso da Sistematização da Assistência de Enfermagem (SAE). Espera-se que a adesão dessa metodologia seja desenvolvida na atuação com os usuários idosos, para proporcionar um cuidado efetivo e resolutivo. Os profissionais que participaram desse estudo relataram que a SAE pode auxiliar em diferentes contextos de atuação, principalmente na qualificação da assistência, permitindo a criação vínculo.

É fundamental que todos os profissionais da saúde estejam preparados para acolher o idoso e sua família, respeitando todos os significados, facilitando na criação de um vínculo mais profundo para com os idosos, transmitindo confiança como estratégia para humanização da atenção do seu atendimento. Os métodos para humanização do cuidado para o enfermeiro é uma forma de transformar a maneira de acompanhar os idosos, com estratégias para a consolidação de uma prática humanizada da sua assistência (Rodrigues, 2019).

Em meio aos estudos que relataram sobre os métodos utilizados pelos enfermeirospara um cuidado humanizado ao idoso na atenção primária, possibilitou compreender as estratégias para humanizar que oportunizam um atendimento de qualidade. Percebeu-se queos artifícios como a promoção de uma atmosfera de confiança, o respeito sobre as decisõesdos idosos, o acolhimento humanizado, práticas para favorecer o fortalecimento de vínculos, empatia, carinho e respeito, linguagem acessível, a implementação da (SAE) para um cuidado efetivo e resolutivo são pontos essenciais no processo de humanização.

Com isso, foi possível verificar que o acolhimento é um método de trabalho que visa amoldar o serviço de saúde proporcionando ao paciente uma postura de acolher, escutar e atender suas necessidades com resolutividade e responsabilidade, diante da assistência. A assistência humanizada ao idoso torna-se essencial onde o enfermeiro proporciona uma atenção que 
valoriza o diálogo para essa população que torna-se vulnerável devido as doenças, escutando-o com atenção, oferecendo-lhe informações de forma clara e objetiva, solucionando dúvidas e inquietações (Resende \& Dias, 2015).

Categoria 3: Benefícios do cuidado humanizado realizado pelo enfermeiro ao idoso

Elaborar atividades de promoção à saúde com vistas à preservação da capacidade funcional poderá contribuir no processo de envelhecimento ativo e saudável. O Programa Saúde da Família tem um importante papel frente as atividades de promoção à saúde, com definição na reorganização dos serviços de saúde, através de ações de promoção, proteção e recuperação da saúde contínua e integral (Sousa, Golçalves \& Gamba, 2018).

Em Lima, et al. (2014) referem que é fundamental avaliar a humanização na atenção básica de saúde diante das experiências vivenciadas pelos idosos, visto que precisam de um cuidado frequente e individualizado. A humanização em saúde é determinada pelo valor atribuído ao esforço dos profissionais envolvidos, quando promove o protagonismo, a corresponsabilidade, com ênfase na dimensão da subjetividade e da singularidade.

A complexidade das demandas de saúde são apresentadas pelos idosos para responder adequadamente às suas necessidades não somente de prevenção e controle de doenças, mas também da promoção da saúde para um envelhecimento ativo e saudável, permitindo maior autonomia e bem-estar. Os idosos representam uma das populações mais vulneráveis, com isso, espera-se que os serviços de atenção primária à saúde sejam capazes de oferecer serviçosaos idosos considerando suas necessidades específicas e busca pela redução das iniquidades em saúde (Schenker \& Costa, 2019).

Diante do que foi analisado em Rodrigues, et al. (2018) os mesmos enfatizam que as orientações atuais para a população idosa caminham na direção da política do envelhecimento ativo e saudável, envolvendo a promoção da saúde, manutenção da saúde, prevenção de agravos, segurança e participação no social. Nos atendimentos na atenção primária, os enfermeiros entrevistados destacaram que transmitem orientações para os idosos para um envelhecimento com qualidade.

A promoção da saúde por meio do atendimento do enfermeiro, promove mudanças com práticas saudáveis, que deve ser incorporada na assistência contínua de todos os profissionais da saúde, evitando possíveis agravos. As medidas de prevenção que estão na proposta de promoção da saúde distinguem-se em quatro níveis: primário, secundário, terciário e quaternário, onde o enfermeiro desenvolve um papel essencial no acompanhamentode idosos para a promoção, proteção e recuperação da saúde (Penha, 2015).

As implicações para a saúde e a qualidade de vida do idoso, o enfermeiro necessita estar qualificado para identificar alterações ou fatores de risco, realizando o devido planejamento da assistência. Verifica-se que as atividades de recreação e lazer são elementos indispensáveis na vida dos idosos, proporcionando-lhes saúde, interesse pela vida, essasestratégias são utilizadas para que possam esquecer os problemas e refletir positivamente paraum bem-estar físico, mental e social repleto de paz e tranquilidade (Clares, 2016).

No que tange o conteúdo sobre a categoria 3, foi possível verificar que diversos fatorespodem contribuir positivamente na qualidade de vida dessa população, envolvendo as atividades de promoção à saúde permitindo cooperar no processo de envelhecimento ativo e saudável dos idosos. Dados revelam a importância sobre a avaliação do processo de humanização na atenção primária, diante das experiências vivenciadas pelos idosos, promovendo o protagonismo.

Nessa Conjuntura, os dados também demostram que os benefícios vão além da prevenção e controle de doenças, permitindo contribuir na promoção de um envelhecimento ativo e saudável, com maior autonomia e bem-estar. A promoção da saúde promove mudanças com práticas saudáveis, que deve ser incorporada na assistência contínua de todos os profissionais da saúde, evitando possíveis agravos, envolvendo a manutenção da saúde, prevenção de agravos, segurança e participação no social.

A literatura destaca os diversos benefícios para os idosos, o estudo de Dias, et al., (2015) acrescenta que o cuidado humanizado promove o respeito como o acolhimento das necessidades de cada indivíduo, destacando-se o conforto, apoio, 
atenção, segurança, higiene, nutrição, apoio espiritual, assistência biopsicossocial. Compreende-se que o atendimento das suas necessidades contribui positivamente, sendo o respeito o valor essencial na prática da enfermagem profissional para o envelhecimento saudável.

\section{Considerações Finais}

Diante do conteúdo estudado, foi possível compreender a importância da atuação do enfermeiro no cuidado humanizado ao idoso na atenção primária, por ser uma população que desenvolve problemas de saúde com maior frequência torna-se uma população mais vulnerável, a literatura aborda que é essencial políticas de saúde pública para promover melhoras na intenção de proporcionar não somente a prevenção e controle de doenças, mas também em proporcionar um envelhecimento ativo e saudável com medidas de grande importância para a atenção integral na saúde do idoso.

Nessa conjuntura, o estudo permitiu constatar por meio dos relatos, que o enfermeiro éum profissional indispensável no contexto da atenção primária, contribuindo positivamente na equipe multiprofissional para a saúde da população assistida, com ênfase na saúde do idoso. Portanto, esse profissional deve estar qualificado para promover o cuidado humanizado de acordo com os princípios e diretrizes do SUS, bem como, na utilização de métodos que facilitam o contato com essa população, como o acolhimento, empatia, respeito, entre outros, com isso, proporciona diversos benefícios para os idosos, como a criação de vínculo que facilita a atuação do enfermeiro mais segura e eficaz.

Por meio da presente investigação científica, que esse estudo de revisão integrativa seja uma fonte de conhecimentos para os estudantes e profissionais da enfermagem, contribuindo positivamente sobre o cuidado humanizado ao idoso na atenção primária, provocando uma reflexão sobre a importância de promover um cuidado único, utilizando os preceitos da ética e valorização do respeito aos ideias dessa população. Deste modo, quepossa estimular novas pesquisas e atualizar o meio científico sobre essa temática.

\section{Referências}

Amthauer, C., \& Falk, J. W. (2017). Discursos dos profissionais de saúde da família na ótica da assistência à saúde do idoso. Revista Online de Pesquisa Cuidado é fundamental, Rio de Janeiro. 9(1), p. 99-105.

Cabral, J. F., et al. (2019). Avaliação da atenção integral à saúde do idoso na percepção de profissionais. Revista Brasileira em Promoção da Saúde. 32, 1-11.

Clares, J. W. B., et al. (2016). Subconjunto de diagnósticos de enfermagem para idosos na Atenção Primária à Saúde. Revista da Escola de Enfermagem da USP, São Paulo. 50(2), 272-278.

Dias, E. G., Souza, E. L. S., \& Mishima, S. M. (2016). Influência da enfermagem na adesão do idoso ao tratamento da hipertensão. Revista Eletrônica Gestão \& Saúde, Rio de Janeiro. 7(3), 1156-1172.

Dias, K. C. C. O., et al. (2015). Estratégias para humanizar o cuidado com o idoso hospitalizado:estudo com enfermeiros assistenciais. Revista Pesquisa Cuidado é Fundamental, Rio de Janeiro. 7(1), 1832-1846.

Faller, J. W., Teston, E. F., \& Marcon, S. S. (2018). Estrutura conceptual do envelhecimento emdiferentes etnias. Revista Gaúcha de Enfermagem, Porto Alegre. $39,1-9$.

Ferenhof, H. A., \& Fernandes, R. F. (2016). Desmistificando a revisão de literatura como base para redação científica: método ssf. Revista ACB: Biblioteconomia em Santa Catarina, Florianópolis. 21(3), 2-14.

Freitas, M. A., \& Alvarez, A. M. (2020). Melhores práticas de enfermagem na saúde da pessoaidosa. Revista de Enfermagem UFPE Online, Recife. 14, 111 .

Hoepers, N. J., et al. (2016). Ações do enfermeiro da estratégia saúde da família na promoção doenvelhecimento saudável. Revista Inova Saúde, Criciúma. $5(1), 42-56$

Lima, T. J. V., et al. (2014). Humanização na atenção básica de saúde na percepção de idosos. Saúde eSociedade, São Paulo. 23(1), $265-276$.

Maciel, M. A. S., Silva, P. C. S., \& Oliveira, L. F. (2019). Percepção dos usuários de uma Estratégia de Saúde da Família sobre o Acolhimento. Revista Eletrônica Acervo Saúde. 11(14), 1-7.

Medeiros, C. B., et al. (2018). A perspectiva do usuário na atenção básica sobre o acolhimento ao idoso. Revista Ciência Plural, 4(3), 43-56. 
Nascimento, E. R. P., et al. (2015). Ambiência de uma emergência hospitalar para o cuidado ao idoso: percepção dos profissionais de enfermagem. Escola Anna Nery, Rio de Janeiro. 19(2), 338-342.

Oliveira, K. S. M., et al. (2018). Violência contra idosos: concepções dos profissionais de enfermagem acerca da detecção e prevenção. Revista Gaúcha de Enfermagem, Porto Alegre. 39, 1-9.

Oliveira, P. P., et al. (2018). A humanização da assistência na ótica de profossionais de enfermagem que cuidam de idosos. Investigación en Enfermería: Imagen y Desarrollo, Bogotá. 20(2), 1-13.

Penha, A. A. G., et al. (2015). Tecnologias na promoção da saúde de idosos com doenças crônicas na atenção primária à saúde. Revista de Enfermagem da UFSM, Santa Maria. 5(3), 406-414.

Piccinini, V. M., Costa, A. E. K., \& Pissaia, L. P. (2017). Implantação da Sistematização da Assistência de Enfermagem como meio de qualificação da assistência ao idoso. Revista Brasileira de Ciências do Envelhecimento, Passo Fundo. 14(3), 307-317.

Resende, J. L., et al. (2015). Assistência do enfermeiro ao idoso na estratégia saúde da família. Revista de Enfermagem do Centro Oeste Mineiro, Minas Gerais. 5(3), 1831-1843.

Rissardo, L. K., et al. (2016). Idosos atendidos em unidade de pronto-atendimento por condições sensíveis à atenção primária à saúde. Revista Mineira de Enfermagem, Minas Gerais. 20(9), 1-8.

Rodrigues, A. R. S., et al. (2018). Percepção de enfermeiros sobre o atendimento à pessoa idosa em contexto de atenção primária. Enfermagem Brasil. 17(2), 99-106.

Rodrigues, W. P., et al. (2019). Percepção dos idosos acerca da assistência humanizada de enfermagem frente ao mal de Parkinson. Brazilian Journal of health Review, Curitiba. 2(4), 3421-3430.

Sanguino, G. Z., et al. (2018). O trabalho de enfermagem no cuidado ao idoso hospitalizado:limites e particularidades. Revista de Pesquisa Cuidado é Fundamental, Rio de Janeiro. 10(1), 160-166.

Santos, S. C., et al. (2016). Saúde do idoso: reflexões acerca da integralidade do cuidado. Revista Brasileira de Promoção \& Saúde, Fortaleza. $29(1)$, 118-127.

Silva, C. D., et al. (2018). Representação da violência doméstica contra a mulher: comparação entre discentes de enfermagem, Revista Gaúcha de Enfermagem, Porto Alegre. 39, 1-9.

Silva, D. P. L., et al. (2018). Envelhecimento e velhice: humanização nos cuidados à pessoa idosa na perspectiva dos alunos do curso técnico em enfermagem da Escola Técnica de Saúde de Cajazeiras - ETSC. Brazilian Journal of health Review, Curitiba. 2(1), 389-398.

Schenker, M., \& Costa, D. H. (2019). Avanços e desafios da atenção à saúde da população idosa com doenças crônicas na Atenção Primária à Saúde. Ciência \& Saúde Coletiva. 24(4), 1369-1380.

Sousa, F. J. D., Golçalves, L. H. T., \& Gamba, M. A. (2018). Capacidade funcional de idosos atendidos pelo programa saúde da família em Benevides, Brasil. Revista Cuidarte. 9(2), 2135-2144.

Sulzbach, C. C., Weiller, T. H., \& Dallepiane, L. B. (2020). Acesso à Atenção Primária à Saúde de longevos: perspectiva de profissionais da Saúde da Família de um município do Rio Grande do Sul. Cadernos de Saúde Coletiva, Rio de Janeiro. 28(3), 373-380, 2020. 